concluded that aprotinin use was the factor associated with mortality when comparing data from 1343 aprotinin-treated patients with those from 6776 given EACA and 2029 given neither therapy.

Two aspects may lead the interested reader to question this conclusion. First, the propensity analysis did not include red blood cell transfusion numbers as a factor (transfusion was graded as either yes or no). More worrisome is that a matched-pairs analysis was relegated to the supplementary data available online from the New England Journal of Medicine. In this analysis, which included 1992 patients with comparable risks, aprotinin showed no effects on 30day $(P=.58)$ and 1-year mortalities $(P=$ .36) relative to EACA.

Thus if propensity scoring is achieved by linear regression, and confounding variables known to be associated with adverse outcomes are excluded, then observational studies show aprotinin to be a dangerous drug. Aprotinin is not seen to be dangerous, however, when the analysis is performed with matching or stratification of risk and known confounders are included.

\section{David Royston, FRCA Consultant Anaesthetist Royal Brompton and Harefield NHS Trust Harefield, UK}

\section{References}

1. Sundt TM. The demise of aprotinin: Our share of the blame. J Thorac Cardiovasc Surg. 2008; 135:729-31.

2. Westaby S. Aprotinin: Twenty-five years of claim and counterclaim. J Thorac Cardiovasc Surg. 2008;135:487-91.
3. Mangano DT, Tudor IC, Dietzel C. The risk associated with aprotinin in cardiac surgery. N Engl J Med. 2006;354:353-65.

4. Levenson.ppt \& Cyns.ppt in Cardiovascular and Renal Drugs Advisory Committee (CRDAC) in Joint Session with the Drug Safety and Risk Management Advisory Committee (DSaRM). Slides Found at www.fda. gov/ohrms/dockets/ac/07/slides2007-4316s100-index.htm. Accessed July 5th 2008.

5. Furnary AP, Wu Y, Hiratzka LF, Grunkemeier GL, Page US 3rd. Aprotinin does not increase the risk of renal failure in cardiac surgery patients. Circulation. 2007; 116(11 Suppl):I127-33.

6. Shaw AD, Stafford-Smith M, White WD, Phillips-Bute B, Swaminathan M, Milano C, et al. The effect of aprotinin on outcome after coronary-artery bypass grafting. $N$ Engl $J$ Med. 2008;358:784-93.

doi:10.1016/j.jtcvs.2008.05.023

\section{Reply to the Editor:}

I appreciate Dr Royston's kind comment regarding my editorial. I am quite certain that he agrees with me that we are "worse off without [aprotinin] in our arsenal.” Indeed, I feel this sentiment particularly this evening, as I wait for a call from the operating room to start repair of an acute dissection in an 80-year-old patient who is receiving warfarin 5 years after coronary bypass and aortic valve replacement. I am also sure that he agrees that, in the best of all possible worlds, the risks and benefits determining the use of a drug should pertain to the welfare of the patient as judged by physicians and not to the litigation risks of a pharmaceutical company as judged by lawyers.

I also appreciate Dr Royston's comments regarding bias. The biases of which he speaks have not (entirely) escaped me; all studies have biases. Randomized studies are of necessity biased at entry. Rigid eligibility criteria are necessary to define a population with sufficient precision to permit analysis, and the demands of equipoise encourage inclusion of low-risk patients for whom harm is the least likely-but so is benefit. Consequently, few such studies truly reflect the spectrum of disease that we face in clinical practice. The populations included in observational studies are more representative of practice; however, the bias introduced by the clinical judgments made in the application of a therapy or administration of a drug impose considerable challenges to balanced interpretation, as so beautifully demonstrated in Dr Royston's letter. I could not agree more. As noted in his comments, understanding the appropriate application and interpretation of propensity analysis demands a learned understanding of the methods as well as the aims of the matching. Unfortunately, few of us (certainly not I) are so statistically sophisticated. I know that in this regard Dr Royston can run circles around me. No contest.

In the end, where we differ, it would appear, is regarding just where the rest of the medical community is struggling. What are the real risks associated with aprotinin? Is it "a potentially harmful drug"? If so, what is the magnitude of that risk? Personally, I remain amazed that today (May 23, 2008), with more than 7100 citations now retrievable on a PubMed search for aprotinin, there is still room for debate.

Thoralf M. Sundt, $M D$ Division of Cardiovascular Surgery Mayo Clinic Rochester, Minn doi:10.1016/j.jtcvs.2008.06.005

\title{
Notice of Correction
}

Re: A near-fatal presentation of a bronchogenic cyst compressing the left main coronary artery. $J$ Thorac Cardiovasc Surg. 2008;135:1395-6.

In the above-noted article, Dr Malcom Finlay should have been listed as the second author on the article. Dr Finlay was the admitting physician in the case. He supplied the angiographic image of the left main stem (LMS) compression (acting as operator) and transoesophageal echo (TOE) image, and he also obtained the patient's consent for publication. 